\section{Validity of Cost and Utility Results?}

Sir,

We read with interest the research conducted by Nguyen and colleagues ${ }^{[1]}$ to identify the costeffective agent for treatment of moderately to severely active rheumatoid arthritis (RA) from the US healthcare payer's perspective.

We are perplexed by the reported results for cost and utilities. The model time horizon is set to 5 years; however, the estimated utility exceeds 5 QALYs and is over 10 QALYs for some of the strategies (table V) ${ }^{[1]}$ Can the authors clarify how this calculation was made?

Furthermore, the acquisition cost of methotrexate (MTX) per cycle is $5 \%$ of that for etanercept (table III). ${ }^{[1]}$ In addition the cost of a non-responder (which includes acquisition and administration costs of tocilizumab and extra physician visits) is still lower than that of the cost of etanercept. Can the authors clarify the difference in the total cost of MTX and etanercept strategies (Delta \$US5000 in table V), ${ }^{[1]}$ given that MTX and 'nonresponders' cost less?

We understand from the paper that serious infection costs are also included in the above estimates for the total strategy costs. However, the incidence of serious infections is reported to be similar for both comparators: MTX and etanercept $(\sim 3.5 \%)$ [table S3 in the Supplemental Digital Content $],{ }^{[1]}$ and therefore it is unclear how this could account for the difference in costs between the two strategies. Furthermore, is this similarity in the safety profile of the two agents plausible considering MTX is an oral therapy?

We are grateful for the authors' comments and clarification and are interested to know if any of our remarks affect the study conclusions.

Alex Diamantopoulos, ${ }^{1}$ Laura Sawyer, ${ }^{1}$ Sarika Ogale ${ }^{2}$ and Fred Dejonckheere ${ }^{3}$

1 Symmetron, London, UK

2 Genentech Inc. South San Francisco, San Francisco, CA, USA

3 F. Hoffman-La Roche, Basel, Switzerland

\section{Acknowledgements}

AD and LS are employed by Symmetron, a consulting firm that has received fees and grants from various pharmaceutical companies, including F. Hoffman-La Roche and Genentech Inc. SO is employed by Genentech Inc. South San Francisco. FD is employed by F. Hoffman-La Roche.

\section{Reference}

1. Nguyen CM, Bounthavong M, Mendes MAS, et al. Cost utility of tumour necrosis factor- $\alpha$ inhibitors for rheumatoid arthritis: an application of Bayesian methods for evidence synthesis in a Markov model. Pharmacoeconomics 2012; 30 (7): 575-93

\section{The Authors' Reply}

\section{Dear Editor}

We thank Diamantopoulos and colleagues ${ }^{[1]}$ for taking an active interest in our study ${ }^{[2]}$ and for their careful examination of our work. As with any simulation, there are inherent limitations; however, the comments and observations by Diamantopoulos et al. ${ }^{[1]}$ have identified a specific error in the QALY calculations.

We used a utility score that was referenced to a disease state that is common in rheumatology studies (American College of Rheumatology [ACR]20); however, our estimation did not adjust for the model cycle, which was 3 months. As Diamantopoulos et al. ${ }^{[1]}$ pointed out, the simulation was for a time horizon of 5 years; therefore, any QALYs gained that exceeded 5 years were implausible, or at least unintuitive.

We used the following equation to estimate the QALY gained for an individual patient: ${ }^{[3]}$

$$
\text { QALYs gained }=\sum_{i=1}^{n} p_{i} D_{i} u_{i}
$$

where $p_{i}$ is the transition probability of being in state $i, D_{i}$ is the duration of being in state $i$, and $u_{i}$ is the utility weight assigned to state $i$. In our article, we accidentally calculated a 1-year cycle duration instead of 3 months or 0.25 years. Therefore, a factor of 0.25 should have been applied to the above equation $\left(D_{i}=0.25\right)$. As a result, incremental cost-effectiveness ratios were 